\title{
People in Antonia's Life
}

Domingo, her husband

María, her mother

Hilario, her father, deceased

Francisco, her older brother

Marta, Francisco's wife

Margarita, her eldest half sister (from her mother's first marriage)

Angélica, her youngest half sister (from her mother's first marriage)

Anita, her younger sister

Marcela, her youngest sister

Moises, Marcela's husband

Felipe, her first son

Magdalena, Felipe's wife

Sebastian, her second son

Juana, Sebastian's wife

Mariano, her third son

Alberto, her fourth son

Paulina, her first daughter

Antonio, Paulina's husband

Rosalva, her second daughter

Guadalupe, her best friend

Christine Eber, her comadre from the United States

Carol-Jean McGreevy-Morales, her comadre from the United States

Sally Meisenhelder, a friend from the United States

Nancy Modiano, a friend in Chiapas, deceased

Heather Sinclair, a friend from the United States 
THIS PAGE INTENTIONALLY LEFT BLANK 